\title{
NOTE
}

\section{Adhesion and Disintegration Phenomena on Fractal Agar Gel Surfaces}

\author{
Ayano Kudo ${ }^{1}$, Marika Sato ${ }^{1}$, Haruna Sawaguchi', Jun-ichi Hotta ${ }^{2}$, Hiroyuki Mayama ${ }^{3}$ and \\ Yoshimune Nonomura ${ }^{1 *}$ \\ ${ }^{1}$ Department of Biochemical Engineering, Graduate School of Science and Engineering, Yamagata University. 4-3-16 Jonan, Yonezawa \\ 992-8510, JAPAN \\ ${ }^{2}$ Department of Bio-system Engineering, Graduate School of Science and Engineering, Yamagata University. 4-3-16 Jonan, Yonezawa \\ 992-8510, JAPAN \\ ${ }^{3}$ Department of Chemistry, Asahikawa Medical University. 2-1-1-1 Asahikawa, Hokkaido 078-8510, JAPAN
}

\begin{abstract}
In the present study, mechanical phenomena on fractal agar gel were analyzed to understand the interfacial properties of hydrophilic biosurfaces. The evaluation of adhesion strength between the fractal agar gel surfaces showed that the fractal structure inhibits the adhesion between the agar gel surfaces. In addition, when the disintegration behavior of an agar gel block was observed between fractal agar gel substrates, the rough structure prevented the sliding of an agar gel block. These findings are useful for understanding the biological significance of rough structure on the biological surfaces.
\end{abstract}

Key words: agar gel, fractal structure, adhesion force, disintegration force

\section{INTRODUCTION}

In our body, there exist several hydrophilic fractal surfaces coated with mucus, which contribute either in effective nutritional absorption or in sensitization of the senses ${ }^{1-3)}$. However, the effect of the rough structure on the interfacial phenomena and the biological significance is unclear as direct observations are difficult to ascertain. A previous study focused on preparing agar gels with hierarchical rough surfaces for hydrophilic biosurface modeling ${ }^{4}$. The hierarchical rough structure induced an acceleration phenomenon that induced the spreading of water droplets ${ }^{4)}$, a trapping phenomenon of liquid droplets and solid particles ${ }^{5,6)}$, and an occurrence of Marangoni flow ${ }^{7)}$. Such interfacial phenomena have also occurred within in vivo systems and may affect the transport phenomenon on biological surfaces.

In this study, the disintegration behavior of an agar gel block was observed between fractal agar gel substrates to clarify the effect of hierarchical rough structures on mechanical phenomena (Fig. 1). First, the adhesion strength between the fractal agar gel surfaces was evaluated by a digital force gauge. Secondly, the disintegration processes of an agar gel block between agar gel substrates were ob-
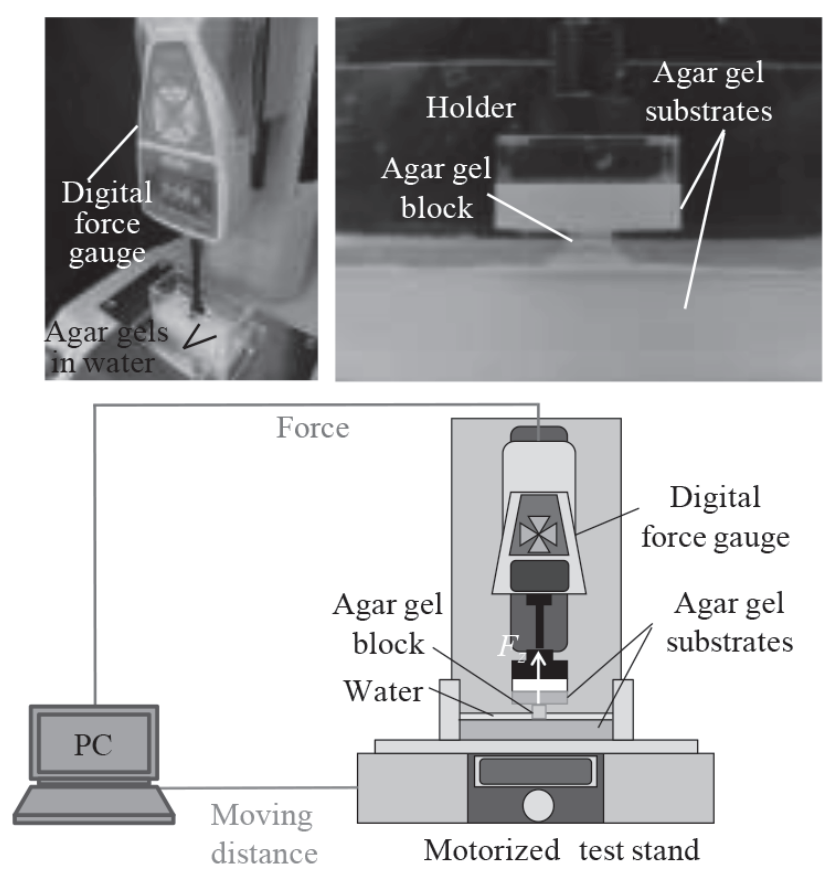

Fig. 1 Images and conceptual diagram of the compression testing system for an agar gel block.

\footnotetext{
*Correspondence to: Yoshimune Nonomura, Department of Biochemical Engineering, Graduate School of Science and Engineering, Yamagata University, 4-3-16 Jonan, Yonezawa 992-8510, JAPAN

E-mail: nonoy@yz.yamagata-u.ac.jp

Accepted July 19, 2016 (received for review April 14, 2016)

Journal of Oleo Science ISSN 1345-8957 print / ISSN 1347-3352 online

http://www.jstage.jst.go.jp/browse/jos/ http://mc.manusriptcentral.com/jjocs
} 
served. Fractal structures may induce significant changes to the contact state and compressional properties.

\section{EXPERIMENTAL PROCEDURES}

Agar powder was purchased from Wako Pure Chemical Industries, Ltd. (Osaka, Japan) and was used without further purification. Rhodamine $6 \mathrm{G}$ and acetone were purchased from Kanto Chemical Co., INC. (Tokyo, Japan). Water was purified by using a DX-15 water deionizing unit (Kurita Water Industries Ltd., Tokyo, Japan). To prepare the flat agar gels, a mixture of agar powder $(6 \mathrm{~g})$ and deionized water $(144 \mathrm{~g})$ was heated and agitated until the agar powder dissolved. The $4 \mathrm{wt} \%$ agar aqueous solution was poured into Petri dishes measuring $10 \mathrm{~cm}$ in diameter. They were left for $12 \mathrm{~h}$ at $277 \mathrm{~K}$ to obtain flat agar gel surfaces. Fractal agar gels were prepared using the fractal plaster, which was transferred into a hierarchical rough surface structure of an alkylketene dimer. The $4 \mathrm{wt} \%$ agar aqueous solutions were poured into Petri dishes, in which the fractal plaster was placed into the center part, and left for $12 \mathrm{~h}$ at $277 \mathrm{~K}$ to obtain fractal agar gels. Box-counting measurements confirmed that the surface structures were fractals with a fractal dimension $D$ of 2.2. The self-similar features were found to hold from $4 \mu \mathrm{m}$ to $30 \mu \mathrm{m}$ of a box size. Agar gel surface geometries were examined using a VK-X100 laser microscope obtained from Keyence(Tokyo, Japan). The roughness was as follows: (1) Flat agar gels exhibited roughness parameters $R_{a}, R_{z}$, and $R_{y}$ of 0.026 , 0.316 , and $0.430 \mu \mathrm{m}$, respectively, and (2) Fractal agar gels displayed $R_{a}, R_{z}$, and $R_{y}$ of $2.14,31.7$, and $35.7 \mu \mathrm{m}$, respectively.

Water film on agar gel surfaces were observed using a fluorescent microscope IX71 (Olympus Corporation, Tokyo, Japan) with an objective lens of $60 \times$ magnification, and a camera (Canon EOS Kiss X5, Canon Inc., Tokyo, Japan). 10 $\mu \mathrm{L}$ of $1 \mu \mathrm{M}$ rhodamine $6 \mathrm{G}$ solution was poured into the center part of the $15 \times 15 \times 5 \mathrm{~mm}^{3}$ fractal or flat agar gel substrates, with the weight of $18.45 \mathrm{~g}$ onto the $24 \times 60 \mathrm{~mm}^{2}$ covered glass.

The compressive stress between two agar gel substrates when the surface was covered with a water film in the depth of $2 \mathrm{~mm}$ (Fig. 1) was evaluated. Figure 1 displays a photograph and a conceptual diagram of a compression testing system. Two agar gel substrates were fixed onto compressional jigs with a digital force gauge ZTA-20N (Imada Co., LTD, Aichi, Japan) that was attached to a vertical motorized test stand EMX-1000N (Imada Co., LTD, Aichi, Japan). The agar gels were applied onto the upper and lower surfaces with a small amount of adhesive agent (Aron alpha, Toagosei Co. Ltd, Tokyo, Japan). The stability between hydrogels and mechanical jigs was checked on each evaluation. The sizes of the upper and lower sub- strates were $20 \times 20 \times 5 \mathrm{~mm}^{3}$ and $30 \times 80 \times 10 \mathrm{~mm}^{3}$, respectively. In Fig. $1, F_{z}$ depicts the vertical force. Specifications of the force gauge are as follows: Maximum working load is $20 \mathrm{~N}$, minimum resolution is $0.01 \mathrm{~N}$, speed adjustment range is $0.5-600 \mathrm{~mm} \mathrm{~min}^{-1}$, and sampling speed is $2000 \mathrm{~s}^{-1}$. The compressive stresses were evaluated when the upper substrates were dropped at $50 \mathrm{~mm} \mathrm{~min}^{-1}$, were kept in contact with the lower substrates for $5 \mathrm{~s}$, and were then risen at $300 \mathrm{~mm} \mathrm{~min}^{-1}$. The disintegration process of an agar gel block containing $1.0 \mathrm{wt} \%$ of an agar powder between agar gel substrates were evaluated when an agar gel block whose size was $5.0 \times 5.0 \times 5.0 \mathrm{~mm}^{3}$ was used. All mechanical evaluations were achieved ten times to check the repeatability. The significance on the effect of fractal structure on the Young's modulus was analyzed. The null hypothesis that Young's modulus values of fractal and flat agar gels are similar each other was tested by conducting a two-sample $t$ test. The null hypothesis was rejected when the $p$-value was above 0.05 .

\section{RESULTS AND DISCUSSION}

In this study, the fractal structure of alkyl ketene dimer (AKD) surfaces was transferred to agar gel substrates. AKD undergoes fractal growth when it solidifies, thus forming super water-repellent fractal surfaces spontaneously $^{8)}$. Figure 2 displays the fluorescent images of the water film on the fractal and flat agar gel substrates. On the fractal agar gel surface, partial bright regions were observed in the image, indicating that water was trapped in surface asperities. The data show that there are many asperities that have a scale of several tens of micrometers on the fractal agar gel substrate, whereas there are very few on the flat agar gel substrate. A previous study tested the fractal dimensions, elasticity, and wettability of the fractal agar gel, which were almost similar with those of a real tongue ${ }^{4,9,10)}$.

The initial experiment evaluated the adhesion strength between the fractal agar gel surfaces. Figure 3 displays the stress-strain curves of the fractal and flat agar gel substrates. Concerning the fractal agar gels, the stress increased gradually and attained a maximum value of $32.2 \pm$ $5.7 \mathrm{kPa}$ when the strain was $0.158 \pm 0.024$. During the 5 -s
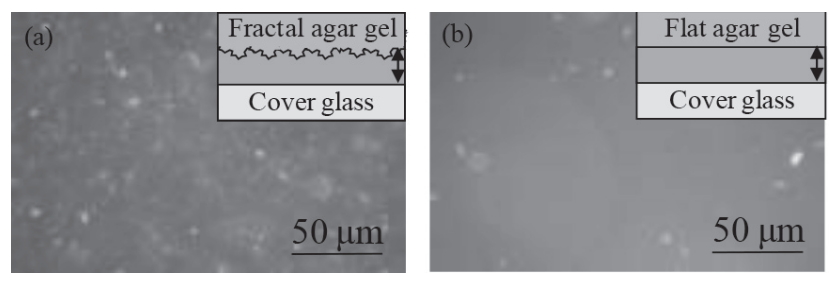

Fig. 2 The fluorescence images of the water film on the fractal (a) and flat agar gel substrates (b) . 


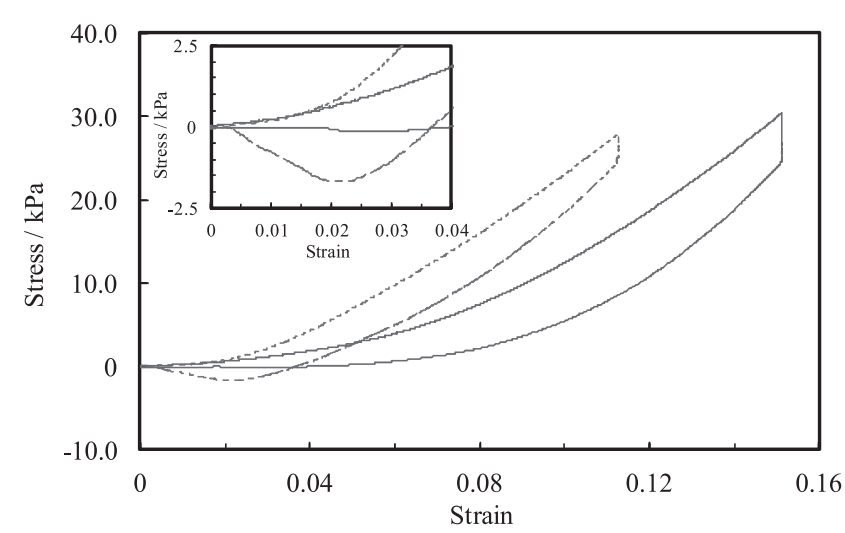

Fig. 3 The stress-strain curves of the fractal and flat agar gel substrates. Straight lines and dotted lines are curves for fractal substrates and flat substrates, respectively.

stop time, the stress was reduced to $25.6 \pm 4.1 \mathrm{kPa}$ due to stress relaxation. Subsequently, the stress was gradually reduced to zero as the strain decreased by raising the upper substrate. Young's modulus, which was determined from the slope following the raise of the upper substrate, was calculated to be $455 \pm 40 \mathrm{kPa}$.

Conversely, a clear hysteresis was observed in the stress-strain curve of the flat agar gels as shown in Fig. 3. The flat agar gel substrate experiment indicated that the stress increased gradually with an increase of strain and attained the maximum value of $27.0 \pm 0.7 \mathrm{kPa}$ at a strain of $0.110 \pm 0.004$. The stress was thereafter reduced to $23.5 \pm$ $0.6 \mathrm{kPa}$ due to the stress relaxation during the stop time of $5 \mathrm{~s}$. This was reduced continuously after moving the upper surface upwards. Interestingly, a significant adhesion force was observed when the strain was smaller than $0.0384 \pm$ 0.0049 . A minimum value of $-1.8 \pm 0.4 \mathrm{kPa}$ was observed at a strain of $0.0219 \pm 0.0077$, whereas the Young's modulus value was calculated as $414 \pm 12 \mathrm{kPa}$. Based on a $t$ test, the significant difference was not recognized between Young's modulus values of fractal and flat agar gel surfaces. These results show that the fractal structure inhibits the adhesion between the agar gel surfaces, although the structure on the agar gel surfaces does not affect the Young's modulus value. In general, the adhesion force is proportional to the real contact area between the substrates ${ }^{11)}$, which is reduced drastically by the fractal surface structure ${ }^{12)}$. The change in the contact area may cause the reduction of the adhesion force.

Thereafter, the disintegration processes of an agar gel block between agar gel substrates were observed (Fig. 1). Regarding the flat agar gel substrate, an agar gel block would not have disintegrated because the block slid out from the inter-space between the agar gel substrates (Fig. $4)$. The block moved horizontally at $1 \mathrm{~s}$ after contact between the block and the substrate and then jumped out after a few seconds. Figure 5 shows the stress-strain curve

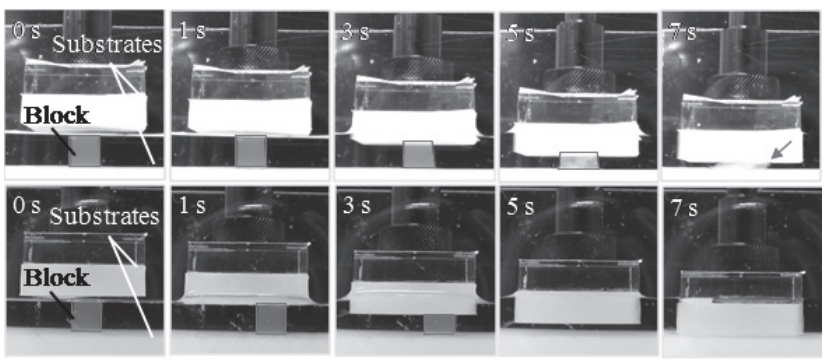

Fig. 4 Images of agar gel blocks between fractal agar gel substrates (top images) and flat agar gel substrates (bottom images).

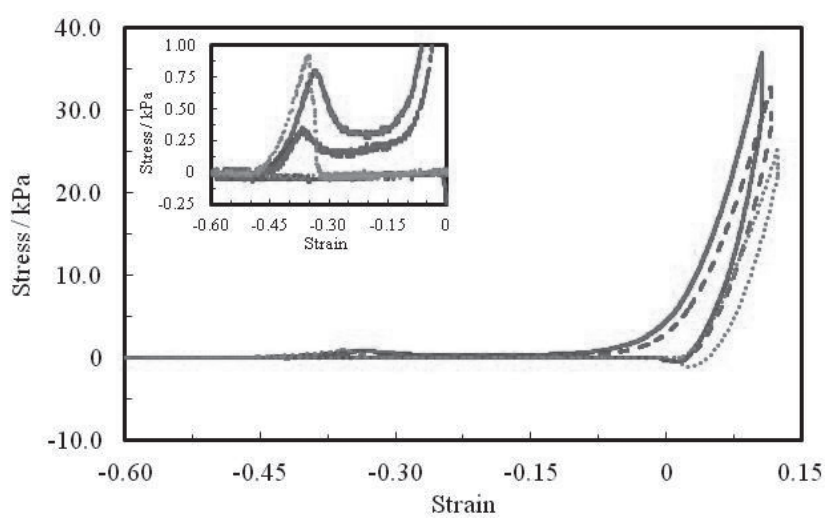

Fig. 5 The stress-strain curve when the block remained at the original position between the fractal agar gel substrates (straight lines), when the block remained at the original position between the flat agar gel substrates (dashed lines), and when the block slid out from the interface between the two flat agar gel substrates (dotted lines).

when the ager gel block was compressed and slid out from the interface between the two flat agar gel substrates. As shown in an inserted figure, the stress increased to $0.994 \pm$ $0.386 \mathrm{kPa}$ and then fell sharply due to the block sliding out. After that, the compression force increased again when the upper agar substrate contacted with the lower substrate. The block did not always slide out from the inter-space between the agar gel substrates. Figure 5 shows the stress-strain curve when the block remained at the original position between the flat agar gel substrates. Stress increased until it reached the rupture stress of $0.450 \pm 0.167$ $\mathrm{kPa}$. The sliding phenomenon was observed in 6 out of 10 repetitions.

In contrast, the remaining phenomena were observed at the interspace between the fractal agar gel substrates. As shown in Fig. 4, after contact with the agar gel substrate, the agar block gradually collapsed over a period of several seconds. Figure 5 displays the stress-strain curve when the block was collapsed at the original position between the fractal agar gel substrates. The stress increased until a rupture stress of $0.810 \pm 0.202 \mathrm{kPa}$ was reached. These 
results indicate that the fractal structure inhibits the sliding behavior of an agar gel block from the interspace between the agar gel substrates. This inhibition may be created by the scratching effect between the fractal agar gel substrate and the agar gel block. The convex on the fractal agar gel can dent into the block and inhibit the sliding effect.

\section{CONCLUSIONS}

Based on some mechanical evaluations, we found some significant adhesion and disintegration phenomena on fractal agar gel surfaces. The adhesion force of $1.8 \pm 0.4$ $\mathrm{kPa}$, which was observed in the case of flat agar gel surfaces, disappeared in the case of fractal agar gel surfaces. While an agar gel block slid out from the interspace between the flat agar gel substrates, the remaining phenomena were observed at the interspace between the fractal agar gel substrates. These mechanical behaviors can be changed by compression speed or any other conditions. Systematic experimental evaluations will be achieved in future. In addition, these interfacial phenomena on hydrophilic fractal surfaces may affect the texture of drinks and gel-type foods. If the tongue surface is flat, a sticky or dancing feeling may be felt when drinking a cup of water or eating a piece of jelly. These findings are useful for understanding the physical origin of food texture and designing novel texture sensors which reflect actual conditions of eating. Hereafter, the relationship between the present mechanical and sensory evaluations will be analyzed.

\section{ACKNOWLEDGMENT}

This study was supported via a Grant-in-Aid for Scientific Research (C) (No. 26390001) from the Ministry of Education, Culture, Sports, Science and Technology, Japan (MEXT).

\section{References}

1) Farman, A.I. Fine structure of the taste bud. J. Ultrastruct. Res. 12, 328-350 (1965).

2) Collins, L.M.C.; Dawes, C. The surface area of the adult human mouth and thickness of the salivary film covering the teeth and oral mucosa. J. Dent. Res., 66, 1300-1302 (1987).

3) Humphrey, S.P.; Williamson, R.T. A review of saliva: normal composition, flow, and function. J. Prosthet. Dent. 85, 162-169(2001).

4) Nonomura, Y.; Morita, Y.; Hikima, T.; Seino, E.; Chida, S.; Mayama, H. Spreading behavior of water droplets on fractal agar gel surfaces. Langmuir 26, 16150$16154(2010)$.

5) Oyama, T.; Mayama, H.; Nonomura, Y. Wetting dynamics of oil-in-water emulsions on agar gel surfaces. Chem. Lett. 42, 871-872 (2013).

6) Seino, E.; Chida, S.; Mayama, H.; Hotta, J.; Nonomura, Y. Wetting dynamics of colloidal dispersions on agar gel surfaces. Colloids Surf. B 122, 1-6(2014).

7) Nonomura, Y.; Chida, S.; Seino, E.; Mayama, H. Anomalous spreading with Marangoni flow on agar gel surfaces. Langmuir 28, 3799-3806 (2012).

8) Onda, T.; Shibuichi, S.; Satoh, N.; Tsujii, K. Super-water-repellent fractal surfaces. Langmuir 12, 21252127(1996).

9) Ranca, H.; Elkhyat, A.; Servais, C.; Mac-Mary, S.; Launay, B.; Humbert, P. Friction coefficient and wettability of oral mucosal tissue: changes induced by a salivary layer. Colloids Surf. A 276, 155-161 (2006).

10) Ishihara, S.; Nakao, S.; Nakauma, M.; Funami, T.; Hori, K.; Ono, T.; Kohyama, K.; Nishinari, K. Compression test of food gels on artificial tongue and its comparison with human test. J. Texture Stud. 44, 104-114(2013).

11) Bharat, B.; Gupta, B.K. Handbook of tribology: materials, coatings, and surface treatments. McGraw-Hill, Inc. (1991)

12) Persson, B.N.J. Theory of rubber friction and contact mechanics. J. Chem. Phys. 115, 3840 (2001). 